\title{
Quantum dot as a spin-current diode: A master-equation approach
}

\author{
Souza, F.M.; Egues, J.C.; Jauho, Antti-Pekka
}

Published in:

Physical Review B Condensed Matter

Link to article, DOI:

10.1103/PhysRevB.75.165303

Publication date:

2007

Document Version

Publisher's PDF, also known as Version of record

Link back to DTU Orbit

Citation (APA):

Souza, F. M., Ėgues, J. C., \& Jauho, A-P. (2007). Quantum dot as a spin-current diode: A master-equation approach. Physical Review B Condensed Matter, 75(16), 165303. https://doi.org/10.1103/PhysRevB.75.165303

\section{General rights}

Copyright and moral rights for the publications made accessible in the public portal are retained by the authors and/or other copyright owners and it is a condition of accessing publications that users recognise and abide by the legal requirements associated with these rights.

- Users may download and print one copy of any publication from the public portal for the purpose of private study or research.

- You may not further distribute the material or use it for any profit-making activity or commercial gain

- You may freely distribute the URL identifying the publication in the public portal 


\title{
Quantum dot as a spin-current diode: A master-equation approach
}

\author{
F. M. Souza, ${ }^{1,2}$ J. C. Egues, ${ }^{3}$ and A. P. Jauho ${ }^{4}$ \\ ${ }^{1}$ Instituto de Estudos Superiores da Amazônia, 66055-260, Belém, Pará, Brazil \\ ${ }^{2}$ Grupo de Física de Materiais da Amazônia, Departamento de Física, Universidade Federal do Pará, 66075-110, Belém, Pará, Brazil \\ ${ }^{3}$ Departamento de Física e Informática, Instituto de Física de São Carlos, \\ Universidade de São Paulo, 13560-970, São Carlos, São Paulo, Brazil \\ ${ }^{4}$ MIC-Department of Micro and Nanotechnology, NanoDTU, Technical University of Denmark, Ørsteds Plads, \\ Bldg. 345E, DK-2800 Kgs. Lyngby, Denmark
}

(Received 10 November 2006; revised manuscript received 19 January 2007; published 5 April 2007)

\begin{abstract}
We report a study of spin-dependent transport in a system composed of a quantum dot coupled to a normal metal lead and a ferromagnetic lead (NM-QD-FM). We use the master equation approach to calculate the spin-resolved currents in the presence of an external bias and an intradot Coulomb interaction. We find that for a range of positive external biases (current flow from the normal metal to the ferromagnet) the current polarization $\wp=\left(I_{\uparrow}-I_{\downarrow}\right) /\left(I_{\uparrow}+I_{\downarrow}\right)$ is suppressed to zero, while for the corresponding negative biases (current flow from the ferromagnet to the normal metal) $\wp$ attains a relative maximum value. The system thus operates as a rectifier for spin-current polarization. This effect follows from an interplay between Coulomb interaction and nonequilibrium spin accumulation in the dot. In the parameter range considered, we also show that the above results can be obtained via nonequilibrium Green functions within a Hartree-Fock type approximation.
\end{abstract}

DOI: 10.1103/PhysRevB.75.165303

PACS number(s): 73.23.Hk

\section{INTRODUCTION}

Polarized transport in spin-dependent nanostructures is a subject of intense study in the emerging field of spintronics, ${ }^{1}$ due to its relevance to the development of spin-based devices. ${ }^{2-4}$ In addition, transport through QDs provides information about fundamental physical phenomena in spin-dependent and strongly correlated systems, such as the Kondo effect, ${ }^{5-9}$ the Coulomb- and spin-blockade effects, ${ }^{10-14}$ spin valve effect and tunneling magnetoresistance (TMR), ${ }^{15-26}$ etc. Spin filters and pumps have also been proposed using QDs coupled to normal metal leads. ${ }^{27-29} \mathrm{~A}$ system of particular interest in this context comprises a quantum dot or a metallic grain coupled to ferromagnetic leads. The ferromagnetism of the leads introduces spin-dependent tunneling rates between the leads and the central region. This results in a nonzero net spin in the central region for asymmetric magnetization geometries. This effect is called spin accumulation or spin imbalance. ${ }^{30-32}$ It has been shown that spin accumulation affects several transport properties, such as magnetoresistance, ${ }^{18,35}$ (negative) differential resistance $^{10,35}$ and the zero-bias anomaly. ${ }^{8,11,18}$ In addition it provides a way to generate and control the current spin polarization via gates or bias voltages, ${ }^{33,34}$ which is one of the main goals within spintronics.

Systems composed of a nonmagnetic lead and a ferromagnetic lead with a quantum dot or a quantum wire as spacer have been analyzed recently. It was pointed out that if the spacer is a dot and the ferromagnetic lead is half-metallic, a mesoscopic current-diode effect arises., ${ }^{4,35,36}$ Spin-current rectification was also predicted in an asymmetric system composed of a ferromagnetic ( $\mathrm{Fe}$ or $\mathrm{Ni}$ ) and nonmagnetic (Au or Pd) contacts coupled to each other via a molecular wire. ${ }^{37}$ Additionally, it was pointed out that a NM-QD-FM system can operate as a spin-filter and as a spin-diode. ${ }^{38}$ In Ref. 38 the authors use the bias voltage to change the reso- nance position of the dot level with respect to the spin-split density of states of the ferromagnetic lead. This gives rise to spin-dependent currents.

Here we study spin-resolved currents in a single-level quantum dot attached to a nonmagnetic lead ("left lead") and to a ferromagnetic lead ("right lead"), Fig. 1. As we shall show, the magnetic asymmetry between the left and right terminals results in a rectification of the current polarization for a particular bias range for which the single electron channel $\epsilon_{d}$ is on-resonance, and the double-occupancy channel $\epsilon_{d}+U$ is off-resonance. More precisely, when the nonmagnetic lead operates as an emitter and the ferromagnetic lead as a collector, defined as the positive bias $(e V>0)$, the current is unpolarized. In contrast, when the ferromagnetic lead is the emitter and the nonmagnetic lead is the collector (negative bias) a spin-polarized current arises. Importantly, this rectification occurs only in this particular bias range, as we shall demonstrate both analytically and numerically. This is attributed to an interplay between nonequilibrium spin accumulation and Coulomb interaction within the dot. For high enough bias voltages, the current polarization is essentially

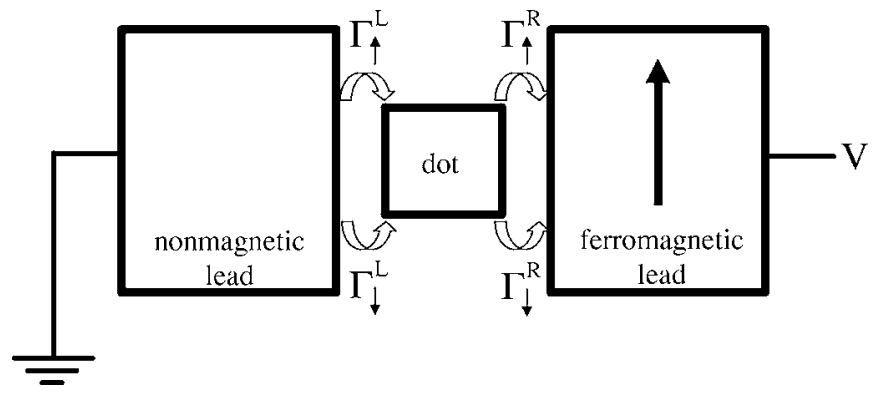

FIG. 1. System studied: a nonmagnetic quantum dot coupled via tunneling barriers to a nonmagnetic left lead and a ferromagnetic right lead. A bias voltage $V$ is applied across the system so that the left $\left(\mu_{L}\right)$ and right $\left(\mu_{R}\right)$ chemical potentials differ by $\mu_{L}-\mu_{R}=e V$. 
symmetric with respect to the bias, and no rectification is found.

In the main body of the paper we employ the masterequation approach of Glazman and Matveev ${ }^{39}$ to describe the spin-dependent transport through the NM-QD-FM junction in the sequential tunneling regime $\left(\Gamma_{0} \ll k_{B} T,{ }^{39}\right.$ where $\Gamma_{0}$ is a characteristic tunneling rate). An alternative description in terms of nonequilibrium Green functions is also presented in the Appendix, that corroborates our results obtained via the master equation.

\section{MODEL AND MASTER EQUATION APPROACH}

The NM-QD-FM system we study is schematically illustrated in Fig. 1. An external bias voltage $V$ drives the system away from equilibrium thus imposing a chemical potential imbalance between the left $(L)$ and the right $(R)$ leads, $\mu_{L}$ $-\mu_{R}=e V$, where $\mu_{L(R)}$ is the chemical potential of the lead $L(R)$ and $e$ is the absolute value of the electron charge (e $>0)$. The system Hamiltonian is

$$
\begin{aligned}
H= & \sum_{\mathbf{k} \sigma \eta} \epsilon_{\mathbf{k} \sigma \eta} c_{\mathbf{k} \sigma \eta}^{\dagger} c_{\mathbf{k} \sigma \eta}+\sum_{\sigma} \epsilon_{d} d_{\sigma}^{\dagger} d_{\sigma}+U d_{\uparrow}^{\dagger} d_{\uparrow} d_{\downarrow}^{\dagger} d_{\downarrow} \\
& +\sum_{\mathbf{k} \sigma \eta}\left(t_{\mathbf{k} \sigma \eta} c_{\mathbf{k} \eta}^{\dagger} d_{\sigma}+t_{\mathbf{k} \eta}^{*} d_{\sigma}^{\dagger} c_{\mathbf{k} \sigma \eta}\right),
\end{aligned}
$$

where $\epsilon_{\mathbf{k} \sigma \eta}$ is the free-electron energy with wave vector $\mathbf{k}$ and spin $\sigma$ in lead $\eta(\eta=L, R), \epsilon_{d}$ is the spin-degenerate dot level, $U$ is the on-site Coulomb repulsion and the operators $c_{\mathbf{k} \sigma \eta}\left(c_{\mathbf{k} \sigma \eta}^{\dagger}\right)$ and $d_{\sigma}\left(d_{\sigma}^{\dagger}\right)$ destroy (create) an electron with spin $\sigma$ in the lead $\eta$ and in the dot, respectively. The matrix element $t_{\mathbf{k} \sigma \eta}$ gives the lead-dot coupling. We do not consider any spin-flip processes.

To calculate the current we use rate equations, ${ }^{24,39}$ which yield

$$
\begin{aligned}
I_{\sigma}^{\eta}= & e\left[\Gamma_{01 \sigma}^{\eta}\left(1-n_{\sigma}-n_{\bar{\sigma}}+n_{\uparrow \downarrow}\right)-\Gamma_{10 \sigma}^{\eta}\left(n_{\sigma}-n_{\uparrow \downarrow}\right)\right] \\
& +e\left[\widetilde{\Gamma}_{01 \sigma}^{\eta}\left(n_{\bar{\sigma}}-n_{\uparrow \downarrow}\right)-\widetilde{\Gamma}_{10 \sigma}^{\eta} n_{\uparrow \downarrow}\right],
\end{aligned}
$$

where we have assumed $\hbar=1$. The parameter $\Gamma_{01 \sigma}^{\eta}$ corresponds to the rate of adding one electron to the dot coming from lead $\eta$, and $\Gamma_{10 \sigma}^{\eta}$ is the rate of moving one electron from the dot to lead $\eta$. In addition, $\widetilde{\Gamma}_{01 \sigma}^{\eta}$ and $\widetilde{\Gamma}_{10 \sigma}^{\eta}$ give the rates of moving one electron with spin $\sigma$ to and from the dot, respectively, when it is already occupied by one electron with opposite spin. Following Ref. 24 we define $n_{\sigma}=\left\langle\hat{n}_{\sigma}\right\rangle$ and $n_{\uparrow \downarrow}$ $=\left\langle\hat{n}_{\uparrow} \hat{n}_{\downarrow}\right\rangle\left(\hat{n}_{\sigma}=d_{\sigma}^{\dagger} d_{\sigma}\right)$ as the dot single and double average occupancies, respectively. The tunneling rates are

$$
\begin{aligned}
& \Gamma_{01 \sigma}^{\eta}=\Gamma_{\sigma}^{\eta} f_{\eta}, \\
& \Gamma_{10 \sigma}^{\eta}=\Gamma_{\sigma}^{\eta}\left(1-f_{\eta}\right), \\
& \widetilde{\Gamma}_{01 \sigma}^{\eta}=\widetilde{\Gamma}_{\sigma}^{\eta} \tilde{f}_{\eta}, \\
& \widetilde{\Gamma}_{10 \sigma}^{\eta}=\widetilde{\Gamma}_{\sigma}^{\eta}\left(1-\tilde{f}_{\eta}\right),
\end{aligned}
$$

where $f_{\eta}=1 /\left\{\exp \left[\left(\epsilon_{d}-\mu_{\eta}\right) /\left(k_{B} T\right)\right]+1\right\}$ and $\tilde{f}_{\eta}=1 /\left\{\exp \left[\left(\epsilon_{d}\right.\right.\right.$ $\left.\left.\left.+U-\mu_{\eta}\right) /\left(k_{B} T\right)\right]+1\right\}$. The rates $\Gamma_{\sigma}^{\eta}$ and $\widetilde{\Gamma}_{\sigma}^{\eta}$ are related to the spin-resolved density of states of lead $\eta$ via $\Gamma_{\sigma}^{\eta}$ $=2 \pi|t|^{2} \rho_{\sigma \eta}\left(\epsilon_{d}\right)$ and $\widetilde{\Gamma}_{\sigma}^{\eta}=2 \pi|t|^{2} \rho_{\sigma \eta}\left(\epsilon_{d}+U\right)$. Here we assume $\Gamma_{\uparrow}^{L}=\Gamma_{\downarrow}^{L}$ and $\Gamma_{\uparrow}^{R} \neq \Gamma_{\downarrow}^{R}$. This reflects the fact that the density of states of the left lead is spin-degenerate while the right one is spin-split. Assuming a constant density of states and a constant tunneling parameter $t$, we have $\Gamma_{\sigma}^{\eta}=\widetilde{\Gamma}_{\sigma}^{\eta}$. With this assumption the terms with $n_{\uparrow \downarrow}$ in Eq. (2) cancel out, ${ }^{24}$ and one simply finds

$$
I_{\sigma}^{\eta}=e\left[\Gamma_{01 \sigma}^{\eta}\left(1-n_{\sigma}-n_{\bar{\sigma}}\right)-\Gamma_{10 \sigma}^{\eta} n_{\sigma}+\widetilde{\Gamma}_{01 \sigma}^{\eta} n_{\bar{\sigma}}\right] .
$$

To calculate the current via Eq. (7) we need to find $n_{\sigma}$ from $^{24}$

$$
\begin{aligned}
\frac{d}{d t} n_{\sigma}= & \Gamma_{01 \sigma}\left(1-n_{\sigma}-n_{\bar{\sigma}}+n_{\uparrow \downarrow}\right)-\Gamma_{10 \sigma}\left(n_{\sigma}-n_{\uparrow \downarrow}\right) \\
& +\widetilde{\Gamma}_{01 \sigma}\left(n_{\bar{\sigma}}-n_{\uparrow \downarrow}\right)-\widetilde{\Gamma}_{10 \sigma} n_{\uparrow \downarrow},
\end{aligned}
$$

where

$$
\begin{aligned}
& \Gamma_{01 \sigma}=\Gamma_{01 \sigma}^{L}+\Gamma_{01 \sigma}^{R}=\Gamma_{\sigma \sigma}^{L} f_{L}+\Gamma_{\sigma}^{R} f_{R}, \\
& \Gamma_{10 \sigma}=\Gamma_{10 \sigma}^{L}+\Gamma_{10 \sigma}^{R}=\Gamma_{\sigma}^{L}\left(1-f_{L}\right)+\Gamma_{\sigma}^{R}\left(1-f_{R}\right), \\
& \widetilde{\Gamma}_{01 \sigma}=\widetilde{\Gamma}_{01 \sigma}^{L}+\widetilde{\Gamma}_{01 \sigma}^{R}=\widetilde{\Gamma}_{\sigma}^{L} \tilde{f}_{L}+\widetilde{\Gamma}_{\sigma}^{R} \widetilde{f}_{R}, \\
& \widetilde{\Gamma}_{10 \sigma}=\widetilde{\Gamma}_{10 \sigma}^{L}+\widetilde{\Gamma}_{10 \sigma}^{R}=\widetilde{\Gamma}_{\sigma}^{L}\left(1-\widetilde{f}_{L}\right)+\widetilde{\Gamma}_{\sigma}^{R}\left(1-\widetilde{f}_{R}\right) .
\end{aligned}
$$

When $\Gamma_{\sigma}^{\eta}=\widetilde{\Gamma}_{\sigma}^{\eta}$, Eq. (8) becomes

$$
\frac{d}{d t} n_{\sigma}=\Gamma_{01 \sigma}\left(1-n_{\sigma}-n_{\bar{\sigma}}\right)-\Gamma_{10 \sigma} n_{\sigma}+\widetilde{\Gamma}_{01 \sigma} n_{\bar{\sigma}},
$$

where the terms with $n_{\uparrow \downarrow}$ cancel out.

Stationary regime. In this regime $\left(d n_{\sigma} / d t=0\right)$ Eq. (13) reduces to

$$
n_{\sigma}=\frac{\Gamma_{01 \sigma}+\left(\widetilde{\Gamma}_{01 \sigma}-\Gamma_{01 \sigma}\right) n_{\bar{\sigma}}}{\Gamma_{01 \sigma}+\Gamma_{10 \sigma}},
$$

which can be solved for each spin component, thus resulting in

$$
n_{\sigma}=\frac{\Gamma_{01 \sigma} \Gamma_{10 \bar{\sigma}}+\Gamma_{01 \bar{\sigma}} \widetilde{\Gamma}_{01 \sigma}}{\Pi_{\sigma}}
$$

where $\quad \Pi_{\sigma}=\left(\Gamma_{01 \sigma}+\Gamma_{10 \sigma}\right)\left(\Gamma_{01 \bar{\sigma}}+\Gamma_{10 \bar{\sigma}}\right)-\left(\widetilde{\Gamma}_{01 \sigma}-\Gamma_{01 \sigma}\right)\left(\widetilde{\Gamma}_{01 \bar{\sigma}}\right.$ $\left.-\Gamma_{01 \bar{\sigma}}\right)$. Using Eq. (15) in Eq. (7) we obtain 


$$
I_{\sigma}^{\eta}=e \frac{\Gamma_{01 \sigma}^{\eta}\left(\Gamma_{10 \sigma} \Gamma_{10 \bar{\sigma}}-\widetilde{\Gamma}_{01 \sigma} \widetilde{\Gamma}_{01 \bar{\sigma}}\right)-\Gamma_{10 \sigma}^{\eta}\left(\Gamma_{01 \sigma} \Gamma_{10 \bar{\sigma}}+\Gamma_{01 \bar{\sigma}} \widetilde{\Gamma}_{01 \sigma}\right)+\widetilde{\Gamma}_{01 \sigma}^{\eta}\left(\Gamma_{01 \bar{\sigma}} \Gamma_{10 \sigma}+\Gamma_{01 \sigma} \widetilde{\Gamma}_{01 \bar{\sigma}}\right)}{\Pi_{\sigma}} .
$$

From Eq. (16) we can readily evaluate the current polarization $\wp=\left(I_{\uparrow}-I_{\downarrow}\right) /\left(I_{\uparrow}+I_{\downarrow}\right)$. Next (Sec. III) we provide some simple analytical results valid when double occupancy is energetically forbidden. Numerical results are presented in Sec. IV.

\section{REGIME OF SINGLY OCCUPIED DOT}

As we shall see, the most interesting behavior takes place when the channel $\epsilon_{d}$ is completely within the conduction window and $\epsilon_{d}+U$ is far above the Fermi energy of the emitter. With this channel configuration we approximate $\tilde{f}_{\eta}=0$, and $f_{L}=1, f_{R}=0$ for $e V>0$ and $f_{L}=0, f_{R}=1$ for $e V<0$. Using this into the occupation and current equations, Eqs. (15) and (16), we find analytical expressions for the first plateau that appears in the current and its polarization for both positive and negative bias voltage. Equation (15) then becomes

$$
n_{\sigma}=\frac{\Gamma_{\sigma}^{\eta} \Gamma_{\bar{\sigma}}^{\bar{\eta}}}{\Gamma_{\sigma}^{\bar{\eta}} \Gamma_{\bar{\sigma}}^{\bar{\eta}}+\Gamma_{\sigma}^{L} \Gamma_{\bar{\sigma}}^{R}+\Gamma_{\bar{\sigma}}^{L} \Gamma_{\sigma}^{R},}
$$

where $\eta=L, \bar{\eta}=R$ for $e V>0$ and $\eta=R, \bar{\eta}=L$ for $e V<0$. The current of the left lead then becomes

$$
I_{\sigma}^{L}= \pm e \frac{\Gamma_{\bar{\sigma}}^{\eta} \Gamma_{\sigma}^{L} \Gamma_{\sigma}^{R}}{\Gamma_{\sigma}^{\eta} \Gamma_{\bar{\sigma}}^{\eta}+\Gamma_{\sigma}^{L} \Gamma_{\bar{\sigma}}^{R}+\Gamma_{\bar{\sigma}}^{L} \Gamma_{\sigma}^{R},}
$$

where $\eta=R$ and the + sign corresponds to $e V>0$, while $\eta$ $=L$ and the - sign to $e V<0$. The right-hand side current is simply given by $I_{\sigma}^{R}=-I_{\sigma}^{L}$ for a spin-conserving stationary regime. Equation (18) gives the (bias-independent) current in the regime addressed here. For the particular case of spinindependent tunneling rates, i.e., $\Gamma_{\sigma}^{L}=\Gamma^{L}$ and $\Gamma_{\sigma}^{R}=\Gamma^{R}$, we obtain

$$
I^{L}=I_{\uparrow}^{L}+I_{\downarrow}^{L}=\left\{\begin{array}{cc}
2 e\left(\Gamma^{L} \Gamma^{R}\right) /\left(2 \Gamma^{L}+\Gamma^{R}\right), & e V>0, \\
-2 e\left(\Gamma^{L} \Gamma^{R}\right) /\left(\Gamma^{L}+2 \Gamma^{R}\right), & e V<0,
\end{array}\right.
$$

in accordance with results already known in the literature. ${ }^{39,40}$

Using Eq. (18) into the definition $\wp=\left(I_{\uparrow}-I_{\downarrow}\right) /\left(I_{\uparrow}+I_{\downarrow}\right)$, we obtain the current polarization plateau

$$
\wp=\frac{\left(\Gamma_{\uparrow}^{\eta}-\Gamma_{\downarrow}^{\eta}\right)}{\left(\Gamma_{\uparrow}^{\eta}+\Gamma_{\downarrow}^{\eta}\right)},
$$

where $\eta=L$ for $e V>0$ and $\eta=R$ for $e V<0$. We model the tunneling rates by $\Gamma_{\uparrow}^{L}=\Gamma_{\downarrow}^{L}=\Gamma_{0}$ and $\Gamma_{\uparrow(\downarrow)}^{R}=\Gamma_{0}(1 \pm p)$, where $p$ $\in[0,1]$ is the spin polarization degree of the ferromagnetic right lead ${ }^{24}$ and $\Gamma_{0}$ the lead-dot coupling. Within this model Eq. (20) gives

$$
\wp= \begin{cases}0, & e V>0, \\ p, & e V<0 .\end{cases}
$$

Thus, when only the level $\epsilon_{d}$ is within the conduction window, the current becomes unpolarized for positive bias, while spin-polarized for negative bias. Therefore the NM-QD-FM junction functions as a current-polarization diode.

\section{RESULTS}

\section{A. Parameters}

We assume that the dot level depends on the bias voltage according to $\epsilon_{d}=\epsilon_{\text {gate }}-x e V$, where $x$ accounts for asymmetric voltage drops along the left and right tunnel barriers. ${ }^{23,24} \epsilon_{\text {gate }}$ can be controlled via gate voltages. For the numerics we take $\epsilon_{\text {gate }}=0.5 \mathrm{meV}, \mu_{L}=0, \mu_{R}=-e V, k_{B} T=212 \mu \mathrm{eV}, U=3 \mathrm{meV}$, and $\Gamma_{0}=10 \mu \mathrm{eV} .^{41,42}$ In Secs. IV B-IV D we assume a symmetric potential drop across the system with $x=0.5$. In Sec. IV E we briefly discuss the asymmetric case with $x \neq 0.5$.

\section{B. Current polarization}

Figure 2 shows the current polarization as a function of the external bias $e V$. We observe that for positive bias the current polarization decreases for increasing bias, reaching zero around $e V=4 \mathrm{meV}$. Conversely, for the negative biases we obtain a maximum polarization $p$ around $e V=-4 \mathrm{meV}$, confirming the analytical result found in Sec. III, Eq. (21). The voltage range for this behavior scales with the parameter $U$. For high enough bias voltages $(|e V| \geqslant 7 \mathrm{meV})$ the polarization reaches the same nonzero plateaus for both positive and negative voltages. Both the suppression $(e V>0)$ and the enhancement $(e V<0)$ of the current polarization are due to the interplay of Coulomb interaction and spin accumulation in the quantum dot. Quite interestingly this interplay affects

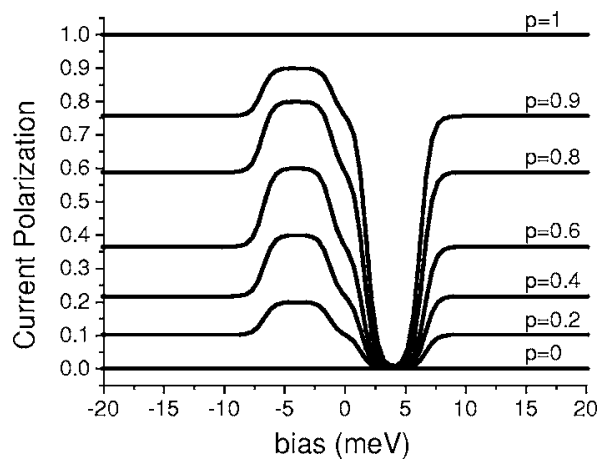

FIG. 2. Current polarization $\wp$ as a function of the external bias. For $p$ values between 0.2 and $0.9, \wp$ reaches zero for some particular positive bias range, while for the negative counterpart it reaches maximum plateaus. 


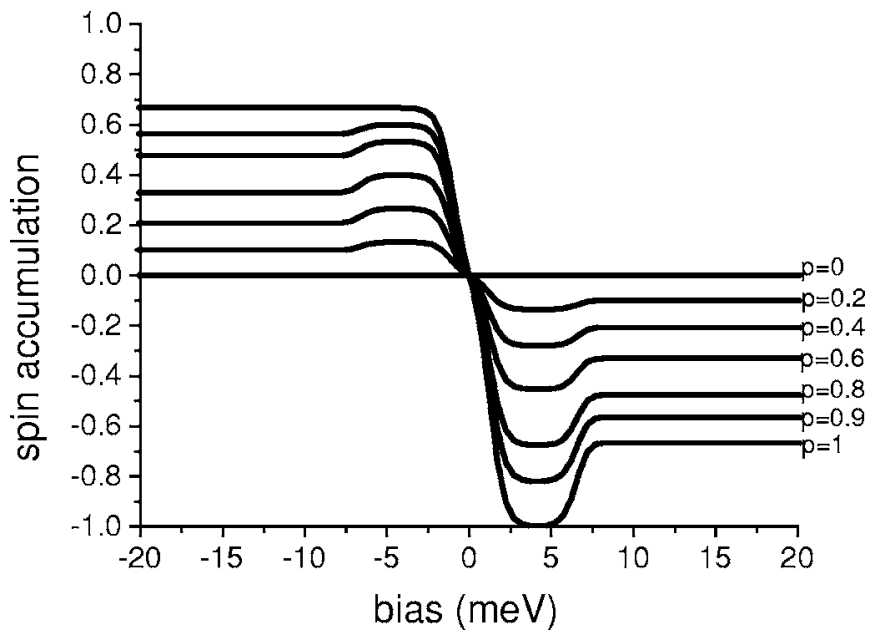

FIG. 3. Spin accumulation $m=n_{\uparrow}-n_{\downarrow}$ as a function of the external bias. For $p=0$ (unpolarized lead) there is no spin accumulation in the dot. When $p$ increases the spin accumulation increases as well becoming negative for $e V>0$ and positive for $e V<0$.

$\wp$ differently with the bias sign, namely, for direct bias it suppresses $\wp$ while for reverse bias it enhances $\wp .{ }^{43}$ The suppression of $\wp$ for positive bias results in the zero polarization seen for all $p$ values except $p=1$. In the half-metallic case $(p=1)$, there is only spin-up current flowing in the system $\left(I_{\uparrow}^{\eta} \neq 0, I_{\downarrow}^{\eta}=0\right)$, so the polarization becomes simply $\wp$ $=\left(I_{\uparrow}^{\eta}-I_{\downarrow}^{\eta}\right) /\left(I_{\uparrow}^{\eta}+I_{\downarrow}^{\eta}\right)=I_{\uparrow}^{\eta} / I_{\uparrow}^{\eta}=1$. On the other hand, for negative bias, the maximum polarization plateau changes as $p$ varies. In particular, $\wp$ attains a plateau equal to the polarization degree of the ferromagnetic lead, according to Eq. (21). To gain a more detailed understanding of the spin-diode effect we investigate next the spin accumulation $m=n_{\uparrow}-n_{\downarrow}$ and the spin-resolved $I-V$ curves as a function of the bias.

\section{Spin accumulation}

Figure 3 shows the spin accumulation $m=n_{\uparrow}-n_{\downarrow}$ as a function of the bias voltage, for distinct polarization parameters $p$. For all the $p$ values considered here we note that $m<0$ for positive bias and $m>0$ for negative bias. This spin-imbalance can be understood in terms of the tunneling rates $\Gamma_{\sigma}^{\eta}$ between dot and leads. Due to the ferromagnetism of the right lead, the rates $\Gamma_{\sigma}^{L}$ and $\Gamma_{\sigma}^{R}$ become asymmetric. For example, for $p=0.2$ the rates are $\Gamma_{\uparrow}^{R}=12 \mu \mathrm{eV}, \Gamma_{\downarrow}^{R}$ $=8 \mu \mathrm{eV}$, and $\Gamma_{\uparrow}^{L}=\Gamma_{\downarrow}^{L}=10 \mu \mathrm{eV}$. For positive bias, $\Gamma_{\sigma}^{L}$ becomes the ingoing tunneling rate for electrons with spin $\sigma$ and $\Gamma_{\sigma}^{R}$ the outgoing tunneling rate. Due to the inequality $\Gamma_{\uparrow}^{R}>\Gamma_{\uparrow}^{L}$, the spin-up electrons can tunnel out the dot faster than they come into it. On the other hand, since $\Gamma_{\downarrow}^{R}<\Gamma_{\downarrow}^{L}$, the spin-down electrons leave the dot slower than they come into it. So on average the spin-down electrons spend more time in the dot than the spin-up ones for $e V>0$, thus $n_{\downarrow}>n_{\uparrow} \Rightarrow m$ $<0$. A similar reasoning applies to the other $p$ values, except for $p=0$ for which there is no accumulation. For negative bias, $\Gamma_{\uparrow}^{L}$ and $\Gamma_{\downarrow}^{L}$ are the outgoing tunneling rates while $\Gamma_{\uparrow}^{R}$ and $\Gamma_{\downarrow}^{R}$ become the ingoing tunneling rates. As a consequence of this interchange, the spin accumulation inverts its sign $(m$
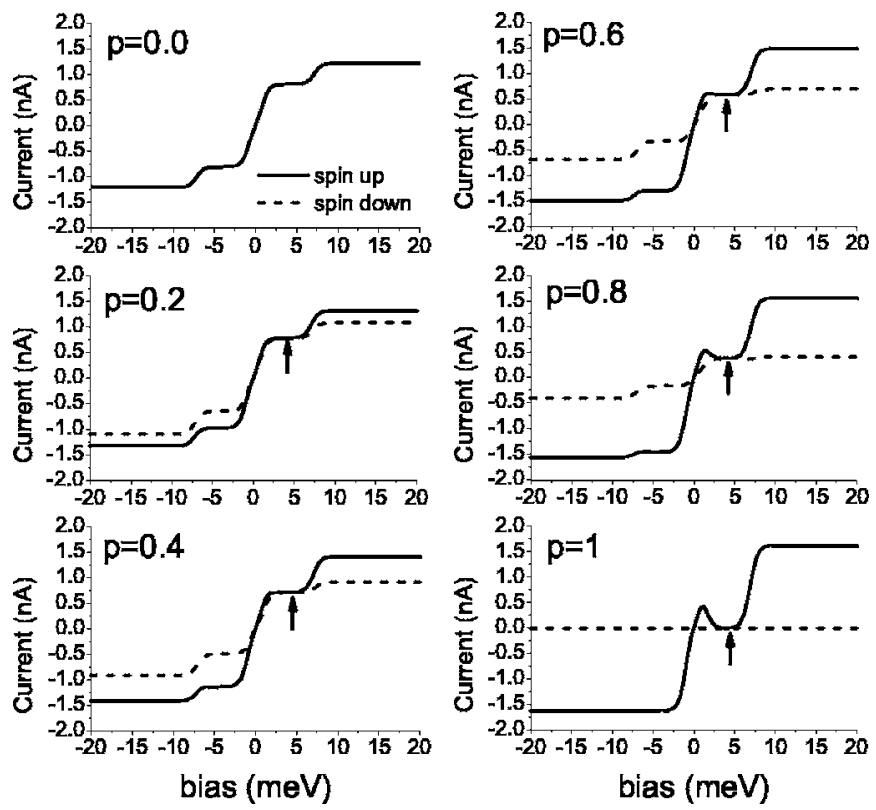

FIG. 4. Spin-resolved currents against bias voltage. For the plateaus indicated by arrows, $I_{\uparrow}$ lies on top of $I_{\downarrow}$. This gives rise to the $\wp=0$ plateau seen in Fig. 2. For big enough polarizations (e.g., $p$ $>0.8)$ a negative-differential resistance range arises in the spin-up current.

$>0$ ). For small $p$ values the spin accumulation is essentially an odd function of the bias, Fig. 3 .

When $p$ increases, though, the imbalance becomes stronger for positive bias. In particular for $p=1, m$ reaches -1 in the positive bias range corresponding to single occupancy $\left(\epsilon_{d}+U>\mu_{L}\right)$, and a constant plateau for all negative bias. This happens because no spin-down states are available in the right lead for $p=1$, so a spin-down electron that enters the dot, coming from the left-hand side $(e V>0)$, cannot leave the dot to the right-hand side. Hence a spin-up electron cannot hop into the dot when $\epsilon_{d}+U>\mu_{L}$, so the accumulation becomes completely spin-down polarized for positive bias. For high enough bias voltages an additional electron with opposite spin can jump into the dot (for both positive and negative bias), thus resulting in a suppression (in modulus) of $m$.

\section{Spin-resolved currents}

In Fig. 4 we show the spin-resolved currents $I_{\uparrow}$ and $I_{\downarrow}$ as a function of the bias voltage for differing polarization parameters $p$. We observe that for positive bias the spin-up and spin-down currents coincide in the plateaus indicated by arrows for any $p$ value. This results in the zero current polarization seen in Fig. 2. In the second plateau, though, $I_{\uparrow}$ attains higher values compared to $I_{\downarrow}$, which enhances $\wp$. The strong suppression of $I_{\uparrow}$ in the first plateau $(e V>0)$ is attributed to the spin imbalance $m<0$ observed for the corresponding bias range (see Fig. 3). More specifically, since the dot is predominantly spin-down occupied for positive bias, the spin-up electrons tend to be more blocked than the spindown ones, thus reducing further $I_{\uparrow}$ and interestingly locking 

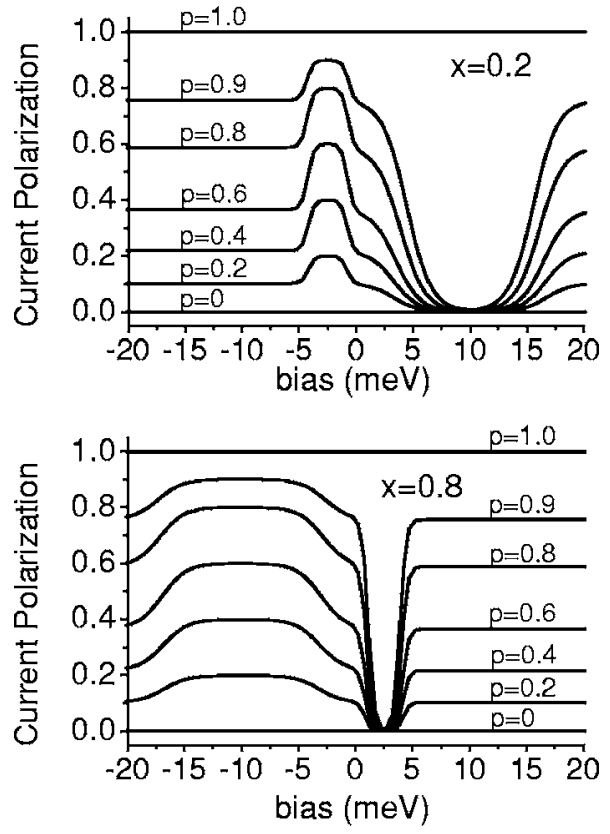

FIG. 5. Current polarization $\wp$ as a function of the bias voltage for the asymmetry parameters $x=0.2$ (upper panel) and $x=0.8$ (bottom panel). We observe that the $\wp=0$ plateau enlarges for $x=0.2$ and shrinks for $x=0.8$ when compared to the $x=0.5$ case. In contrast, the maximum plateau $\wp=p$ reduces for $x=0.2$ and enlarges for $x=0.8$ when compared to the $x=0.5$ case.

it on top of $I_{\downarrow}$. In contrast, for negative bias we have the population inversion $m>0$. This gives a stronger suppression of $I_{\downarrow}$ as compared to $I_{\uparrow}$, which enhances the difference between $I_{\uparrow}$ and $I_{\downarrow}$, and consequently $\wp$. When the channel $\epsilon_{d}+U$ reaches resonance $(e V \lesssim-7 \mathrm{meV})$ both the $I_{\uparrow}$ and $I_{\downarrow}$ plateaus attain values somewhat closer to each other, thus reducing the current polarization (see Fig. 2).

In particular for $p=1$ the $I_{\downarrow}$ is zero for any bias voltage since there are no spin-down states available in the righthand lead. The $I_{\uparrow}$ increases slightly (for positive bias) while the dot is becoming populated. When the population is high enough the Coulomb interaction plays a role and the spin-up current goes down to zero. ${ }^{44}$ This gives rise to a negative differential conductance at the beginning of the first plateau for $e V>0$ (see Fig. 4 with $p=1$ ). ${ }^{45}$ For negative bias (and $p=1) I_{\uparrow}$ attains one plateau instead of two steps as for the other $p$ values. This is expected because the spin-down electrons do not participate in the transport in this case, so no Coulomb interaction effect arises. Note that for $p=1$ the system can operate as a mesoscopic current diode. , $35,36^{-36}$

\section{E. Effects of the bias-drop asymmetry}

Here we consider the effects of an asymmetric bias drop, i.e., $x \neq 0.5$. As Fig. 5 shows, the asymmetry in the bias drop gives rise to quantitative, but not qualitative changes. For $x$ $=0.2$ the current polarization $\wp$ goes to zero much slower with the bias than it does for $x=0.5$. This is so because the resonance condition $\epsilon_{d} \leqslant \mu_{L}(e V>0)$, which is necessary to have $\wp=0$ (see Sec. III), happens for higher bias when $x$ decreases. For negative bias the resonance $\epsilon_{d} \leqslant \mu_{R}$ is reached faster (i.e., at lower biases as compared with the $x=0.5$ case) for decreasing $x$. This, in turn, translates into a steeper enhancement of $\wp$ which then attains a plateau at $\wp=p$ [see Eq. (21)]. In addition, for $x=0.2$ the zero current-polarization plateau $(e V>0)$ enlarges while the maximum plateau $(\mathrm{eV}$ $<0)$ shrinks compared to the respective $x=0.5$ widths. For $x=0.8$ the resonance $\epsilon_{d} \leqslant \mu_{L}(e V>0)$ takes place faster with the bias when compared to the $x=0.2$ and $x=0.5$ cases. This results in the steeper suppression of $\wp$ and the shrinkage of the zero current-polarization bias range. For negative biases the resonance condition $\epsilon_{d} \leqslant \mu_{R}$ for $x=0.8$ is more slowly attained with the bias as compared to the $x=0.2$ and $x=0.5$ cases. Consequently, the polarization $\wp$ reaches the plateau at $\wp=p$ for higher bias voltages (in modulus).

\section{CONCLUSION}

We propose a NM-QD-FM system which operates as a diode for the current polarization. More specifically, when double occupancy is forbidden in the system, i.e., the channel $\epsilon_{d}+U$ is far above the chemical potential of the emitter lead, the system carries an unpolarized current for positive bias and a spin-polarized current for negative bias. This effect is a result of the interplay between spin accumulation in the dot and the Coulomb interaction. Interestingly, for positive biases the spin-resolved currents $I_{\uparrow}$ and $I_{\downarrow}$ lock onto the same plateau for a particular bias range, thus resulting in $\wp$ $=0$.

\section{ACKNOWLEDGMENTS}

The authors acknowledge G. Platero, K. Flensberg, T. Novotný, and J. P. Morten for helpful comments. Two of the authors (F.M.S.) and (J.C.E.) acknowledge the kind hospitality at the Centre for Advanced Study (Oslo) during the revision stage of this work. One of the authors (J.C.E.) acknowledges support from CNPq and FAPESP.

\section{APPENDIX: NONEQUILIBRIUM GREEN FUNCTIONS}

In the main text we have formulated the problem via the master equation formalism. Here we show that Eqs. (17) and (18), from which our main result Eq. (21) directly follows, can be obtained via the Keldysh formalism. We start with the well-known equation for the stationary current ${ }^{46,47}$

$$
I_{\sigma}^{\eta}=i e \int \frac{d \epsilon}{2 \pi}\left\{\Gamma_{\sigma}^{\eta}\left\{\left[G_{\sigma \sigma}^{r}(\epsilon)-G_{\sigma \sigma}^{a}(\epsilon)\right] f_{\eta}(\epsilon)+G_{\sigma \sigma}^{<}(\epsilon)\right\}\right\},
$$

where $G_{\sigma \sigma}^{r}, G_{\sigma \sigma}^{a}$, and $G_{\sigma \sigma}^{<}$are the retarded, advanced, and lesser Green functions, respectively. To calculate these we apply the equation of motion technique and use the HartreeFock approximation to factorize high-order correlation functions in the resulting chain of equations. ${ }^{48}$ The retarded Green function becomes

$$
G_{\sigma \sigma}^{r}(\epsilon)=\frac{1}{g_{\sigma \sigma}^{-1}(\epsilon)-\Sigma_{\sigma}^{r}(\epsilon)},
$$

where $\Sigma_{\sigma}^{r}(\epsilon)$ is the noninteracting tunneling self-energy given in the wide band approximation by $\Sigma_{\sigma}^{r}(\epsilon)$ 
$=-i \Gamma_{\sigma} / 2=-i\left(\Gamma_{\sigma}^{L}+\Gamma_{\sigma}^{R}\right) / 2$, and $g_{\sigma \sigma}(\epsilon)$ is the dot Green function without coupling to leads,

$$
g_{\sigma \sigma}(\epsilon)=\frac{\epsilon-\epsilon_{d}-U\left(1-n_{\bar{\sigma}}\right)}{\left(\epsilon-\epsilon_{d}\right)\left(\epsilon-\epsilon_{d}-U\right)},
$$

where $n_{\bar{\sigma}}$ is the dot occupation number, with $\bar{\sigma}=-\sigma$. This occupation can be calculated self-consistently via

$$
n_{\sigma}=\left\langle d_{\sigma}^{\dagger} d_{\sigma}\right\rangle=-i \int \frac{d \epsilon}{2 \pi} G_{\sigma \sigma}^{<}(\epsilon),
$$

where the correlation function $G_{\sigma \sigma}^{<}(\epsilon)$ is given by the Keldysh equation

$$
G_{\sigma \sigma}^{<}(\epsilon)=G_{\sigma \sigma}^{r}(\epsilon) \Sigma_{\sigma}^{<} G_{\sigma \sigma}^{a}(\epsilon) .
$$

The advanced Green function $G_{\sigma \sigma}^{a}(\epsilon)$ is given by $G_{\sigma \sigma}^{a}(\epsilon)$ $=\left[G_{\sigma \sigma}^{r}(\epsilon)\right]^{*}$, while $\Sigma_{\sigma}^{<}=i\left(\Gamma_{\sigma}^{L} f_{L}+\Gamma_{\sigma}^{R} f_{R}\right)$. In order to consider the same channel configuration adopted in Sec. III, we assume a large $U\left(\epsilon_{d}+U \gg \mu_{\eta}\right)$ so the retarded Green function becomes

$$
G_{\sigma \sigma}^{r}(\epsilon)=\frac{\left(1-n_{\bar{\sigma}}\right)}{\epsilon-\epsilon_{d}-\Sigma_{\sigma}^{r}\left(1-n_{\bar{\sigma}}\right)},
$$

and the lesser Green function reads

$$
G_{\sigma \sigma}^{<}(\epsilon)=\frac{i\left(\Gamma_{\sigma}^{L} f_{L}+\Gamma_{\sigma}^{R} f_{R}\right)\left(1-n_{\bar{\sigma}}\right)^{2}}{\left(\epsilon-\epsilon_{d}\right)^{2}+\left(\frac{\Gamma_{\sigma}}{2}\right)^{2}\left(1-n_{\bar{\sigma}}\right)^{2}} .
$$

Substituting Eq. (A7) into Eq. (A4) we have

$$
n_{\sigma}=\frac{\left(1-n_{\bar{\sigma}}\right)^{2}}{2 \pi} \int d \epsilon \frac{\left(\Gamma_{\sigma}^{L} f_{L}+\Gamma_{\sigma}^{R} f_{R}\right)}{\left(\epsilon-\epsilon_{d}\right)^{2}+\left(\frac{\Gamma_{\sigma}}{2}\right)^{2}\left(1-n_{\bar{\sigma}}\right)^{2}}
$$

Now assuming that the dot level $\epsilon_{d}$ is completely on resonance within the conduction window between $\mu_{L}$ and $\mu_{R}$ for positive or negative bias ${ }^{49}$ we can integrate Eq. (A8) in order to obtain

$$
n_{\sigma}=\frac{\Gamma_{\sigma}^{\eta}}{\Gamma_{\sigma}}\left(1-n_{\bar{\sigma}}\right)
$$

where $\eta=L$ for $e V>0$ or $\eta=R$ for $e V<0$. Solving Eq. (A9) for each spin component we find exactly Eq. (17).

To obtain Eq. (18) from the Green functions, we substitute Eqs. (A6) and (A7) into the current formula (A1), which gives

$$
I_{\sigma}^{L}=e \int \frac{d \epsilon}{2 \pi} \frac{\Gamma_{\sigma}^{L} \Gamma_{\sigma}^{R}\left(1-n_{\bar{\sigma}}\right)^{2}\left(f_{L}-f_{R}\right)}{\left(\epsilon-\epsilon_{d}\right)^{2}+\left(\frac{\Gamma_{\sigma}}{2}\right)^{2}\left(1-n_{\bar{\sigma}}\right)^{2}} .
$$

Solving Eq. (A10) with the same assumptions adopted previously ${ }^{49}$ we find

$$
I_{\sigma}^{L}= \pm e \frac{\Gamma_{\sigma}^{L} \Gamma_{\sigma}^{R}}{\Gamma_{\sigma}}\left(1-n_{\bar{\sigma}}\right),
$$

where + and - signs correspond to $e V>0$ and $e V<0$, respectively. Equation (A11) can also be written as Eq. (18).
${ }^{1}$ For an overview see, e.g., Semiconductor Spintronics and Quantum Computation, edited by D. D. Awschalom, D. Loss, and N. Samarth (Springer, Berlin, 2002); I. Zutic, J. Fabian, and S. Das Sarma, Rev. Mod. Phys. 76, 323 (2004).

${ }^{2}$ D. Loss and D. P. DiVincenzo, Phys. Rev. A 57, 120 (1998).

${ }^{3}$ H.-A. Engel, P. Recher, and D. Loss, Solid State Commun. 119, 229 (2001).

${ }^{4}$ M. Wilczyński, R. Świrkowicz, W. Rudziński, J. Barnaś, and V. Dugaev, J. Magn. Magn. Mater. 290-291, 209 (2005).

${ }^{5}$ R. Świrkowicz, M. Wilczyński, M. Wawrzyniak, and J. Barnaś, Phys. Rev. B 73, 193312 (2006).

${ }^{6}$ J. Martinek, M. Sindel, L. Borda, J. Barnaś, R. Bulla, J. König, G. Schön, S. Maekawa, and J. von Delft, Phys. Rev. B 72, 121302(R) (2005).

${ }^{7}$ Y. Utsumi, J. Martinek, G. Schön, H. Imamura, and S. Maekawa, Phys. Rev. B 71, 245116 (2005).

${ }^{8}$ J. Martinek, Y. Utsumi, H. Imamura, J. Barnaś, S. Maekawa, J. König, and G. Schön, Phys. Rev. Lett. 91, 127203 (2003).

${ }^{9}$ P. Zhang, Q.-K. Xue, Y. Wang, and X. C. Xie, Phys. Rev. Lett. 89, 286803 (2002).

${ }^{10}$ F. Elste and C. Timm, Phys. Rev. B 73, 235305 (2006).

${ }^{11}$ I. Weymann, J. Barnaś, J. König, J. Martinek, and G. Schön, Phys. Rev. B 72, 113301 (2005).

${ }^{12}$ A. Cottet, W. Belzig, and C. Bruder, Phys. Rev. Lett. 92, 206801
(2004); A. Cottet and W. Belzig, Europhys. Lett. 66, 405 (2004).

${ }^{13}$ J. Barnaś and A. Fert, Phys. Rev. Lett. 80, 1058 (1998).

${ }^{14}$ S. Takahashi and S. Maekawa, Phys. Rev. Lett. 80, 1758 (1998).

${ }^{15}$ K. Walczak and G. Platero, Cent. Eur. J. Phys. 4, 30 (2006).

${ }^{16}$ I. Weymann and J. Barnaś, Phys. Rev. B 73, 033409 (2006).

${ }^{17}$ J. Varalda, A. J. A. de Oliveira, D. H. Mosca, J.-M. George, M. Eddrief, M. Marangolo, and V. H. Etgens, Phys. Rev. B 72, 081302(R) (2005).

${ }^{18}$ I. Weymann, J. König, J. Martinek, J. Barnaś, and G. Schön, Phys. Rev. B 72, 115334 (2005).

${ }^{19}$ F. M. Souza, J. C. Egues, and A. P. Jauho, Braz. J. Phys. 34, 565 (2004).

${ }^{20}$ F. M. Souza, J. C. Egues, and A. P. Jauho, in Proceedings of the 26th International Conference on the Physics of Semiconductors (ICPS 26), Edinburgh, 2002, edited by J. H. Davies and A. R. Long. See cond-mat/0209263.

${ }^{21}$ R. López and D. Sánchez, Phys. Rev. Lett. 90, 116602 (2003).

${ }^{22}$ I. Weymann and J. Barnaś, Phys. Status Solidi B 236, 651 (2003).

${ }^{23}$ W. Rudziński, J. Barnaś, and M. Jankowska, J. Magn. Magn. Mater. 261, 319 (2003).

${ }^{24}$ W. Rudziński and J. Barnaś, Phys. Rev. B 64, 085318 (2001).

${ }^{25}$ J. König and J. Martinek, Phys. Rev. Lett. 90, 166602 (2003).

${ }^{26}$ M. Braun, J. König, and J. Martinek, Phys. Rev. B 70, 195345 
(2004).

${ }^{27}$ P. Recher, E. V. Sukhorukov, and D. Loss, Phys. Rev. Lett. 85, 1962 (2000).

${ }^{28}$ H.-A. Engel and D. Loss, Phys. Rev. B 65, 195321 (2002).

${ }^{29}$ E. Cota, R. Aguado, and G. Platero, Phys. Rev. Lett. 94, 107202 (2005).

${ }^{30}$ A. Brataas, Y. V. Nazarov, J. Inoue, and G. E. W. Bauer, Phys. Rev. B 59, 93 (1999).

${ }^{31}$ H. Imamura, S. Takahashi, and S. Maekawa, Phys. Rev. B 59, 6017 (1999).

${ }^{32}$ J. Martinek, J. Barnaś, S. Maekawa, H. Schoeller, and G. Schön, Phys. Rev. B 66, 014402 (2002).

${ }^{33}$ J. Wang, K. S. Chan, and D. Y. Xing, Phys. Rev. B 72, 115311 (2005).

${ }^{34}$ W. Kuo and C. D. Chen, Phys. Rev. B 65, 104427 (2002).

${ }^{35}$ I. Weymann and J. Barnaś, Phys. Rev. B 73, 205309 (2006).

${ }^{36}$ R. Świrkowicz, J. Barnaś, M. Wilczyński, W. Rudziński, and V. K. Dugaev, J. Magn. Magn. Mater. 272-276, 1959 (2004).

${ }^{37}$ H. Dalgleish and G. Kirczenow, Phys. Rev. B 73, 235436 (2006).

${ }^{38}$ A. A. Shokri, M. Mardaani, and K. Esfarjani, Physica E (Amsterdam) 27, 325 (2005).

${ }^{39}$ L. I. Glazman and K. A. Matveev, JETP Lett. 48, 445 (1988).

${ }^{40}$ Y. V. Nazarov and J. J. R. Struben, Phys. Rev. B 53, 15466 (1996); A. Thielmann, M. H. Hettler, J. König, and G. Schön, ibid. 68, 115105 (2003).

${ }^{41}$ Typical values of $\Gamma_{0}$ and $U$ can be found in, for example, A. Kogan, S. Amasha, D. Goldhaber-Gordon, G. Granger, M. A. Kastner, and H. Shtrikman, Phys. Rev. Lett. 93, 166602 (2004); F. Simmel, R. H. Blick, J. P. Kotthaus, W. Wegscheider, and M.
Bichler, ibid. 83, 804 (1999); D. Goldhaber-Gordon, H. Shtrikman, D. Mahalu, D. Abusch-Magder, U. Meirav, and M. A. Kastner, Nature (London) 391, 156 (1998).

${ }^{42}$ In the context of nonequilibrium Green function (see Appendix) we note that by increasing the coupling parameter $\Gamma_{0}$, the width of the dot level increases as well, thus resulting in more smooth curves in Figs. 2-5.

${ }^{43}$ For a system with two ferromagnetic leads coupled to a quantum dot (FM-QD-FM), it is possible to have an enhancement of $\wp$ for negative biases and a suppression of $\wp$ for positive biases when the lead magnetizations are aligned antiparallel. However, the polarization does not vanish for any particular bias range and consequently no rectification effect is observed: F. M. Souza, Ph.D. thesis, University of São Paulo, Brazil, 2004.

${ }^{44}$ A similar bump in the total current $\left(I_{\uparrow}+I_{\downarrow}\right)$ was also seen in B. R. Bulka, Phys. Rev. B 62, 1186 (2000), and also in Refs. 4, 23, and 24.

${ }^{45}$ A negative differential resistance was also seen in J. Fransson, Phys. Rev. B 72, 075314 (2005).

${ }^{46}$ Y. Meir and N. S. Wingreen, Phys. Rev. Lett. 68, 2512 (1992).

${ }^{47}$ A. P. Jauho, N. S. Wingreen, and Y. Meir, Phys. Rev. B 50, 5528 (1994).

${ }^{48}$ H. Haug and A. P. Jauho, Quantum Kinetics in Transport and Optics of Semiconductors, Springer Solid-State Sciences Vol. 123 (Springer, New York, 1996).

${ }^{49}$ More precisely we assume that $\mu_{R}<\epsilon_{d}<\mu_{L}$ for $e V>0$ and $\mu_{L}$ $<\epsilon_{d}<\mu_{R}$ for $e V<0$. In addition we approximate $f_{L} \approx 1$ and $f_{R} \approx 0$ for $e V>0$ and $f_{L} \approx 0$ and $f_{R} \approx 1$ for $e V<0$. 